\title{
Hopf algebra of non-commutative field theory
}

\author{
Adrian Tanasă and Fabien Vignes-Tourneret
}

\begin{abstract}
We construct here the Hopf algebra structure underlying the process of renormalization of non-commutative quantum field theory.
\end{abstract}

Mathematics Subject Classification (2000). 16W30, 81R60, 81T15, 81T18.

Keywords. Quantum field theory, renormalization, non-commutative geometry, Hopf algebra.

\section{Introduction and motivation}

Hopf algebras (see for example [Kas95] or [DNR01]) are today one of the most studied structures in mathematics. In relation with quantum field theories (QFTs), Hopf algebras were proven to be a natural framework for the description of the forest structure of renormalization - the Connes-Kreimer algebras [CK00], [CK01]. Ever since there has been an important amount of work with respect to this new class of Hopf algebras (for a general review see for example [Kre05]).

However, this construction was realized so far only at the level of commutative QFT. When uplifting to non-commutative quantum field theory (NCQFT), the interaction is no longer local. Thus, the vertices of the associated Feynman diagrams can now be represented as in Figure 1.

Recently, NCQFT models were also proven to be renor-

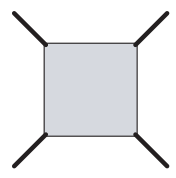
malizable at any order in perturbation theories, despite the ultraviolet-infrared mixing problem. The non-commutative analogous of the Bogoliubov-Parasiuk-Hepp-Zimmerman (BPHZ) theorem was proven for the GrosseWulkenhaar $\Phi^{4}$ scalar model in [GW05a], [GW05b]. In [GMRVT06] a general proof in $x$-space, using multiscale analysis, was given. The parametric representation was implemented for this model in [GR07]. Furthermore, the Mellin representation of the non-commutative Feynman amplitudes was achieved in [GMRT07]. Finally, the dimensional regularization and renormalization were constructed in [GT07].

With respect to the form of the associated propagator, a second class of NCQFT models exists. This second class contains the non-commutative Gross-Neveu and the 
Langmann-Szabo-Zarembo (LSZ) [LSZ04] models. The associate BPHZ theorem was proven in [VT07] for the non-commutative Gross-Neveu model. Moreover, the parametric representation [RT07] and the Mellin representation [GMRT07] were also implemented for this class of models. For a recent review on different issues of renormalizability of NCQFT the interested reader is referred to [Riv07].

Note that even though recent progress has been made in [DGWW07], [GW07], [BGS07], physicists do not yet have a renormalizable non-commutative gauge theory.

In this article we construct the Hopf algebra structure associated to the renormalization of these NCQFT models. The paper is organized as follows. In the next section we give some insights on the renormalization of NCQFT with respect to renormalization of commutative QFT. The third section is devoted to the Hopf algebra structure of Feynman diagrams. In the last section we state and prove our main result.

\section{Renormalization of non-commutative quantum field theory}

In this section we briefly recall some features of both commutative and non-commutative Euclidean renormalization. We will mainly focus on the Grosse-Wulkenhaar model [GW05b], [GMRVT06] or non-commutative $\Phi_{4}^{4}$ theory. It consists in a scalar quantum field theory on the four-dimensional Moyal space. Its action is given by

$$
S[\phi]=\int d^{4} x\left(-\frac{1}{2} \phi(-\Delta) \phi+\frac{\Omega^{2}}{2} \tilde{x}^{2} \phi^{2}+\frac{1}{2} m^{2} \phi^{2}+\frac{\lambda}{4} \phi \star \phi \star \phi \star \phi\right)(x)
$$

with $\tilde{x}_{\mu}=2\left(\Theta^{-1} x\right)_{\mu}$ and $\Theta$ a four-by-four skew-symmetric matrix which encodes the non-commutative character of space time: $\left[x^{\mu}, x^{\nu}\right]_{\star}={ }_{l} \Theta^{\mu \nu}$. It has been shown renormalizable to all orders of perturbation.

Furthermore, as already stated in the previous section, the same renormalization results also hold for the non-commutative Gross-Neveu model [VT07] and the generalized LSZ model [GMRVT06]:

$$
\begin{aligned}
S_{\mathrm{GN}} & =\int d^{2} x\left[\bar{\psi}\left(-i \not \partial+\Omega \not{x}+m+\mu \gamma_{5}\right) \psi-\sum_{A=1}^{3} \frac{g_{A}}{4}\left(\mathcal{g}^{A} \star \mathcal{g}^{A}\right)(x)\right], \\
\mathcal{g}^{A} & =\bar{\psi} \star \Gamma^{A} \psi, \quad \Gamma_{1}=\mathbb{1}, \quad \Gamma_{2}=\gamma^{\mu}, \quad \Gamma_{3}=\gamma_{5}, \\
S_{\mathrm{gLSZ}} & =\int d^{4} x\left[\bar{\phi}\left(\left(-l \partial_{\mu}+\Omega_{1} \tilde{x}_{\mu}\right)^{2}+\Omega_{2} \tilde{x}^{2}+m^{2}\right) \phi+\frac{\lambda}{2} \bar{\phi} \star \phi \star \bar{\phi} \star \phi\right](x) .
\end{aligned}
$$

2.1. Topology and power counting. Let $G$ be a graph with $V$ vertices and $I$ internal lines. Interactions of quantum field theories on the Moyal space are only invariant under cyclic permutation of the incoming/outcoming fields. This restricted invariance replaces the permutation invariance which was present in the case of local interactions. 
A good way to keep track of such a reduced invariance is to draw Feynman graphs as ribbon graphs. Moreover there exists a basis for the Schwartz class functions where the Moyal product becomes an ordinary matrix product [GW03], [GBV88]. This further justifies the ribbon representation.

Let us consider the example of Figure 2. Propagators in a ribbon graph are made of double lines. Let us call $F$ the number of faces (loops made of single lines) of a

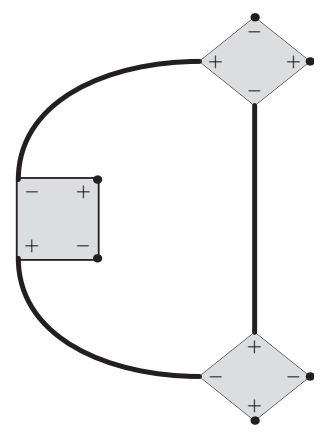

(a) $x$-space representation

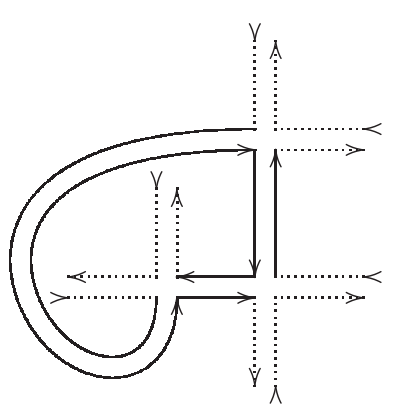

(b) Ribbon representation

Figure 2. A graph with two broken faces.

ribbon graph. The graph of Figure 2 (b) has $V=3, I=3, F=2$. Each ribbon graph can be drawn on a manifold of genus $g$. The genus is computed from the Euler characteristic $\chi=F-I+V=2-2 g$. If $g=0$ one has a planar graph, otherwise one has a non-planar graph. For example, the graph of Figure 2 (b) may be drawn on a manifold of genus 0 . Note that some of the $F$ faces of a graph may be "broken" by external legs. In our example, both faces are broken. We denote the number of broken faces by $B$.

Furthermore let $N$ be the number of external legs of the graph. For the commutative $\phi^{4}$ model one has the following superficial degree of convergence: $\omega=N-4$. Thus one has to deal only with the renormalization of the two- and four-point functions. In the case of the Grosse-Wulkenhaar model the situation is different. In [GW05a], [GW05b], [GR07] it was proven that

$$
\omega=(N-4)+8 g+4(B-1) .
$$

Note that, as shown in [RT07], one has the same power counting for the LSZ-like model (2.4). The one of the Gross-Neveu model (2.2) is more involved but leads to the same conclusion: one has to deal only with the renormalization of the $B=1$, planar two- and four-point graphs, hereafter qualified as planar regular. 
2.2. Locality vs. Moyality. A crucial aspect of the uplifting from commutative to non-commutative renormalization is that the principle of locality of renormalized interactions of commutative QFT is replaced with a new principle: renormalized interactions have a non-local Moyal vertex form. This is nothing but the analog of the locality phenomenon which occurs in commutative renormalization. One can thus speak, in the case of non-commutative renormalization, of a new type of renormalization group, where the locality is just replaced by "Moyality". The divergent parts of the planar regular two- and four-point graphs with one broken face (the only divergent graphs) are proportional to the (1PI) tree level terms of the perturbative expansion. Such a new definition of "locality" was suggested in [Kre05], see equation (62).

Let us also argue here that, despite this uplifting, the combinatorial backbone of renormalization theory is almost the same when dealing with commutative or noncommutative QFT. Thus the combinatorics of non-commutative renormalization will be shown to be encoded by a Hopf algebra.

2.3. Renormalization as a factorization issue. The basic operation for renormalization is the disentanglement of a graph $\Gamma$ into pieces $\gamma$ and cograph $\Gamma / \gamma$. It is exactly this operation that was present at the level of commutative renormalization and that gave rise to a Hopf algebra structure.

We now argue that this factorization process is also present at the level of noncommutative renormalization. Indeed, consider the dimensional renormalization scheme for the Grosse-Wulkenhaar model. The parametric representation constructed in [GR07] writes the Feynman amplitude $\phi(\Gamma)$ as

$$
\phi(\Gamma)=K \int_{0}^{1} \prod_{\ell=1}^{L}\left[d t_{\ell}\left(1-t_{\ell}^{2}\right)^{\frac{D}{2}-1}\right] H U_{G, \bar{V}}(t)^{-\frac{D}{2}} e^{-\frac{H V_{G}}{H U_{G}}},
$$

where $K$ is some constant,

$$
t_{\ell}=\tanh \frac{\alpha_{\ell}}{2}, \quad \ell=1, \ldots, L,
$$

where $\alpha_{\ell}$ are the parameters associated to any of the propagators of the graph. In [GR07] it was furthermore proved that $H U$ and $H V$ are polynomials in the set of variables $t_{\ell}$.

Considering now a primitive divergent subgraph $\gamma$ of $\Gamma$ and rescaling the parameters $t$ of its internal edges, it was shown in [GT07] that

$$
H U_{\Gamma}^{l}=H U_{\gamma}^{l} H U_{\Gamma / \gamma}
$$

where by the index $l$ we understand the leading terms under the rescaling. A similar factorization theorem was also proven for the exponential part in (2.6) of the Feynman amplitude $\phi(\Gamma)$. 
Moreover, in [GMRVT06] an analogous phenomenon of factorization was shown for the Grosse-Wulkenhaar model in position space, namely the planar regular graphs contribute to the renormalization of the mass, wave function, harmonic frequency $\Omega$ and coupling constant; see equation (2.1).

\section{Hopf algebra structure of Feynman diagrams}

3.1. Hopf algebra reminder. In this subsection we recall the general definition of a Hopf algebra (for further details one can refer for example to [Kas95], [DNR01]).

Definition 3.1 (Algebra). A unital associative algebra $\mathcal{A}$ over a field $\mathbb{K}$ is a $\mathbb{K}$-linear space endowed with two algebra homomorphisms:

- a product $m: \mathcal{A} \otimes \mathcal{A} \rightarrow \mathcal{A}$ satisfying the associativity condition

$$
m \circ(m \otimes \mathrm{id})(\Gamma)=m \circ(\mathrm{id} \otimes m)(\Gamma) \quad \text { for all } \Gamma \in \mathcal{A} ;
$$

- a unit $u: \mathbb{K} \rightarrow \mathcal{A}$ satisfying

$$
m \circ(u \otimes \mathrm{id})(\Gamma)=\Gamma=m \circ(\mathrm{id} \otimes u)(\Gamma) \quad \text { for all } \Gamma \in \mathcal{A} .
$$

Definition 3.2 (Coalgebra). A coalgebra $\mathcal{C}$ over a field $\mathbb{K}$ is a $\mathbb{K}$-linear space endowed with two algebra homomorphisms:

- a coproduct $\Delta: \mathscr{C} \rightarrow \mathscr{C} \otimes \mathscr{C}$ satisfying the coassociativity condition:

$$
(\Delta \otimes \mathrm{id}) \circ \Delta(\Gamma)=(\mathrm{id} \otimes \Delta) \circ \Delta(\Gamma) \text { for all } \Gamma \in \mathcal{C} ;
$$

- a counit $\varepsilon: \ell \rightarrow \mathbb{K}$ satisfying

$$
(\varepsilon \otimes \text { id }) \circ \Delta(\Gamma)=\Gamma=(\text { id } \otimes \varepsilon) \circ \Delta(\Gamma) \text { for all } \Gamma \in \mathcal{C} .
$$

Definition 3.3 (Bialgebra). A bialgebra $\mathscr{B}$ over a field $\mathbb{K}$ is a $\mathbb{K}$-linear space endowed with both an algebra and a coalgebra structure (see Definitions 3.1 and 3.2) such that the coproduct and the counit are unital algebra homomorphisms (or, equivalently, the product and unit are coalgebra homomorphisms):

$$
\begin{aligned}
\Delta \circ m_{\mathscr{B}} & =m_{\mathcal{B} \otimes \mathcal{B}} \circ(\Delta \otimes \Delta), & \Delta(\mathbb{1}) & =\mathbb{1} \otimes \mathbb{1}, \\
\varepsilon \circ m_{\mathscr{B}} & =m_{\mathbb{K}} \circ(\varepsilon \otimes \varepsilon), & \varepsilon(\mathbb{1}) & =1 .
\end{aligned}
$$

Definition 3.4 (Graded bialgebra). A graded bialgebra is a bialgebra graded as a linear space,

$$
\mathcal{B}=\bigoplus_{n=0}^{\infty} \mathcal{B}^{(n)},
$$


such that the grading is compatible with the algebra and coalgebra structures:

$$
\mathscr{B}^{(n)} \mathscr{B}^{(m)} \subseteq \mathscr{B}^{(n+m)} \quad \text { and } \quad \Delta \mathscr{B}^{(n)} \subseteq \bigoplus_{k=0}^{n} \mathscr{B}^{(k)} \otimes \mathscr{B}^{(n-k)} .
$$

Definition 3.5 (Connectedness). A connected bialgebra is a graded bialgebra $\mathscr{B}$ for which $\mathscr{B}^{(0)}=u(\mathbb{K})$.

One can then define a Hopf algebra.

Definition 3.6 (Hopf algebra). A Hopf algebra $\mathscr{H}$ over a field $\mathbb{K}$ is a bialgebra over $\mathbb{K}$ equipped with an antipode map $S: \mathscr{H} \rightarrow \mathscr{H}$ satisfying

$$
m \circ(S \otimes \mathrm{id}) \circ \Delta=u \circ \varepsilon=m \circ(\mathrm{id} \otimes S) \circ \Delta .
$$

Finally we remind the reader of a useful lemma.

Lemma 3.1 ([Man03]). Any connected graded bialgebra is a Hopf algebra whose antipode is given by $S(\mathbb{1})=\mathbb{1}$ and recursively by any of the two following formulas for $\Gamma \neq \mathbb{1}$ :

$$
\begin{aligned}
& S(\Gamma)=-\Gamma-\sum_{(\Gamma)} S\left(\Gamma^{\prime}\right) \Gamma^{\prime \prime}, \\
& S(\Gamma)=-\Gamma-\sum_{(\Gamma)} \Gamma^{\prime} S\left(\Gamma^{\prime \prime}\right) .
\end{aligned}
$$

Here we used Sweedler's notation.

3.2. Locality and the residue map. In quantum field theory, Feynman graphs are built from a certain set of edges and vertices $R=R_{E} \cup R_{V}$. This set is given by the particle content of the model and by the type of interactions one wants to consider. For example, in the commutative $\phi_{4}^{4}$ theory (which will be our benchmark until Section 4) $R_{E}$ contains only the scalar bosonic line, while $R_{V}$ contains the local four-point vertex and the two-point vertices corresponding to the mass and wave function renormalization:

$$
R_{E}=\{-\}, \quad\{\chi<, \stackrel{0}{\bullet}, \stackrel{1}{\leftarrow}\} .
$$

In the following we will still write $R_{V}$ for the free algebra generated by the elements of $R_{V}$. Let us now consider the algebra $\mathscr{H}$ generated by a certain class of graphs (connected, 1PI, etc.) made out of the set $R$. 
Definition 3.7 (Subgraph). Let $\Gamma \in \mathscr{H}$, let $\Gamma^{[1]}$ be its set of internal lines and $\Gamma^{[0]}$ its vertices. A subgraph $\gamma$ of $\Gamma$, written $\gamma \subset \Gamma$, consists in a subset $\gamma^{[1]}$ of $\Gamma^{[1]}$ and the vertices of $\Gamma^{[0]}$ hooked to the lines in $\gamma^{[1]}$. Note that with such a definition, $\gamma$ is truncated.

Definition 3.8 (Shrinkable subgraph). Let $\Gamma \in \mathcal{H}$. A subgraph $\emptyset \varsubsetneqq \gamma \varsubsetneqq \Gamma$ is said shrinkable if res $(\gamma) \in R_{V}$. The set of shrinkable subgraphs of $\Gamma$ will be denoted by $\underline{\Gamma}$.

Note that so far we did not really define what the map res is. We now do it. First we assume that it is an algebra homomorphism from $\mathscr{H}$ to $\mathscr{H} \cup R_{V}$. Then to compute the graphical residue of a generator of $\mathscr{H}$, we need the following remarks and definitions.

The coproduct of $\mathscr{H}$ (usually given by (3.15)) drives the combinatorial and algebraic aspects of renormalization if it corresponds to some analytical facts. Before we explain this, let us recall the following definitions.

Definition 3.9. The (unrenormalized) Feynman rules are a homomorphism $\phi$ from $\mathscr{H}$ to $\mathcal{A}$. The precise definition of $\mathcal{A}$ depends on the regularization scheme employed (in dimensional regularization, $\mathcal{A}$ is the Laurent series).

Definition 3.10. The projection $T$ is a map from $\mathcal{A}$ to $\mathcal{A}$ such that

$$
\left(\operatorname{id}_{\mathcal{A}}-T\right) \circ \phi(\Gamma)<\infty
$$

for all $\Gamma \in \mathscr{H}$ and $\Gamma$ primitive. This means that if $\phi(\Gamma)$ is superficially divergent (as the cut-off is removed) then its overall divergence is totally included in $T \circ \phi(\Gamma)$.

External structures. The projection $T$ extracts the divergent part of the amplitude $\phi(\Gamma)$. In the case of a two-point graph this divergent part decomposes into two pieces. The first one is a mass term whereas the second one contributes to the wave function renormalization (recall that the propagator of the commutative $\phi^{4}$ theory is $\left.\left(-\Delta+m^{2}\right)^{-1}\right)$. To distinguish between these two, one introduces external structures [CK00], [Kre05]. It consists of the following endomorphisms of $\mathcal{A}$ (in $x$-space representation):

$$
\begin{aligned}
\left\langle\sigma_{0}, \phi(\Gamma)\right\rangle & =\rho_{0}(\Gamma) \delta_{y}(x) \\
\left\langle\sigma_{1}, \phi(\Gamma)\right\rangle & =\rho_{1}(\Gamma) \Delta \delta_{y}(x) \\
\left\langle\sigma_{2}, \phi(\Gamma)\right\rangle & =\rho_{2}(\Gamma) \delta_{x_{2}}\left(x_{1}\right) \delta_{x_{3}}\left(x_{1}\right) \delta_{x_{4}}\left(x_{1}\right)
\end{aligned}
$$

where the $\rho_{i}$ 's are characters on $\mathcal{A}$. If $K_{\Gamma}$ is the kernel of the amplitude $\phi(\Gamma)$, these characters are given by

$$
\rho_{0}(\Gamma)=\int d^{4} z K_{\Gamma}(x, z),
$$




$$
\begin{aligned}
& \rho_{1}(\Gamma)=\frac{1}{8} \int d^{4} z(z-x)^{2} K_{\Gamma}(x, z), \\
& \rho_{2}(\Gamma)=\int d^{4} x_{2} d^{4} x_{3} d^{4} x_{4} K_{\Gamma}\left(x, x_{2}, x_{3}, x_{4}\right) .
\end{aligned}
$$

Recall that commutative field theories are usually translation invariant so that none of the $\rho_{i}$ 's depend on $x$. With these notations, $T=\sigma_{0}+\sigma_{1}$ on a two-point graph and $T=\sigma_{2}$ on a four-point graph.

There is now a way to relate the analytical operations $\sigma_{i}$ 's to the graphical map res:

Definition 3.11 (Residue). The residue map res: $\mathscr{H} \rightarrow \mathscr{H} \cup R_{V}$ is defined by

$$
\left\langle\sigma_{i}, \phi(\Gamma)\right\rangle=\rho_{i}(\Gamma)\left\langle\sigma_{i}, \phi \circ \operatorname{res}(\Gamma)\right\rangle,
$$

where $i=0$ or 1 for a two-point graph and $i=2$ on a four-point graph.

Following equations (3.11) and (3.12) one finds

$$
\begin{array}{ll}
\phi \circ \operatorname{res}(\Gamma)=\delta_{y}(x)+\Delta \delta_{y}(x) & \text { if } \Gamma \text { is a two-point graph, } \\
\phi \circ \operatorname{res}(\Gamma)=\delta_{x_{2}}\left(x_{1}\right) \delta_{x_{3}}\left(x_{1}\right) \delta_{x_{4}}\left(x_{1}\right) & \text { if } \Gamma \text { is a four-point graph, }
\end{array}
$$

which leads to the following graphical definitions:

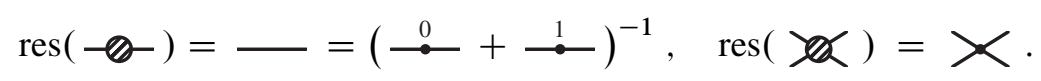

Equation (3.13) means that the divergent part of a graph $\Gamma$ "looks like" another graph called $\operatorname{res}(\Gamma)$. For a renormalizable quantum field theory the residue of any superficially divergent graph belongs to $R_{V}$. This is the usual statement according to which all the divergences of a renormalizable field theory can be "absorbed" in a redefinition of the various coupling constants. If the theory is local then $\operatorname{res}(\Gamma)$ corresponds to the graph obtained from $\Gamma$ by shrinking all its internal lines to a point. But this is a particular case and we have to define res as reflecting the appropriate projection $T$. For example, we will see in the next section that the residue of a non-commutative graph is not a local graph anymore.

The $T$ operation is designed to extract the "main" part of graphs. For the convergent ones there is no good distinction between $T \circ \phi(\Gamma)$ and (id $-T) \circ \phi(\Gamma)$ : both are convergent expressions. That is why $T$ is mainly defined on (superficially) divergent graphs. Nevertheless one can define $T$ to be $\mathrm{id}_{\mathcal{A}}$ on convergent graphs. Condition (3.10) is then trivially fulfilled and equation (3.13) is satisfied with res $=\mathrm{id} \mathscr{H}$ and $\rho$ the trivial character. 
3.3. Coassociative coproducts. Using the definitions of Section 3.2 we have the following lemma.

Lemma 3.2 (Coassociativity). Let $\Gamma \in \mathcal{H}$. Suppose that the following hold:

(1) $\operatorname{res}(\gamma) \in R_{V}, \operatorname{res}\left(\gamma^{\prime}\right) \in R_{V}$ and $\operatorname{res}\left(\gamma / \gamma^{\prime}\right) \in R_{V}$ for all $\gamma \in \underline{\Gamma}$ and for all $\gamma^{\prime} \in \gamma$

(2) for all $\gamma_{1} \in \mathscr{H}$ and for all $\gamma_{2} \in \mathscr{H}$ such that $\operatorname{res}\left(\gamma_{1}\right) \in R_{V}$ and $\operatorname{res}\left(\gamma_{2}\right) \in R_{V}$, there exists gluing data $G$ such that $\operatorname{res}\left(\gamma_{1}{ }^{\circ} G \gamma_{2}\right) \in R_{V}$.

Then the coproduct

is coassociative.

$$
\begin{aligned}
\Delta \Gamma & =\Gamma \otimes \mathbb{1}+\mathbb{1} \otimes \Gamma+\Delta^{\prime} \Gamma, \\
\Delta^{\prime} \Gamma & =\sum_{\gamma \in \underline{\Gamma}} \gamma \otimes \Gamma / \gamma .
\end{aligned}
$$

Observe that $\Gamma / \gamma$ is the graph obtained from $\Gamma$ by replacing $\gamma \subset \Gamma$ with its residue. Then res $(\gamma) \in R_{V}$ implies that $\Gamma / \gamma \in \mathscr{H}$. We prove this lemma by following closely [CK00].

Proof. First note that $(\Delta \otimes$ id $) \Delta=($ id $\otimes \Delta) \Delta \Leftrightarrow\left(\Delta^{\prime} \otimes\right.$ id $) \Delta^{\prime}=\left(\right.$ id $\left.\otimes \Delta^{\prime}\right) \Delta^{\prime}$, which means that all the following subgraphs can be considered as neither full nor empty. Let $\Gamma$ be a generator of $\mathscr{H}$,

$$
\begin{aligned}
\left(\Delta^{\prime} \otimes \text { id }\right) \Delta^{\prime} \Gamma & =\left(\Delta^{\prime} \otimes \text { id }\right) \sum_{\gamma \in \underline{\Gamma}} \gamma \otimes \Gamma / \gamma \\
& =\sum_{\gamma \in \underline{\Gamma}} \sum_{\gamma^{\prime} \in \underline{\gamma}} \gamma^{\prime} \otimes \gamma / \gamma^{\prime} \otimes \Gamma / \gamma, \\
\left(\mathrm{id} \otimes \Delta^{\prime}\right) \Delta^{\prime} \Gamma & =\sum_{\gamma^{\prime} \in \underline{\Gamma}} \sum_{\gamma^{\prime \prime} \in \underline{\Gamma} / \gamma^{\prime}} \gamma^{\prime} \otimes \gamma^{\prime \prime} \otimes\left(\Gamma / \gamma^{\prime}\right) / \gamma^{\prime \prime} .
\end{aligned}
$$

By the Definitions 3.7, 3.8 and 3.11 it is clear that $\gamma^{\prime} \in \gamma$ and $\gamma \in \underline{\Gamma}$ implies $\gamma^{\prime} \in \underline{\Gamma}$. This implicitly uses the fact that the residue of a graph is independent of the surrounding of this graph and really only depends on the graph itself: $\operatorname{res}(\gamma)$ is the same wether $\gamma$ is a subgraph of another graph or not. Equation (3.17) can then be rewritten as

$$
\left(\Delta^{\prime} \otimes \text { id }\right) \Delta^{\prime} \Gamma=\sum_{\gamma^{\prime} \in \underline{\Gamma}} \sum_{\gamma \in \underline{\Gamma} \text { with } \gamma \supsetneqq \gamma^{\prime}} \gamma^{\prime} \otimes \gamma / \gamma^{\prime} \otimes \Gamma / \gamma .
$$

It is now enough to prove equality of (3.18) and (3.19) at fixed $\gamma^{\prime} \in \underline{\Gamma}$. Let us first fix a subgraph $\gamma \in \underline{\Gamma}$ such that $\gamma \supsetneqq \gamma^{\prime}$ and prove that there exists a graph $\gamma^{\prime \prime} \in \underline{\Gamma / \gamma^{\prime}}$ 
such that $\gamma / \gamma^{\prime} \otimes \Gamma / \gamma=\gamma^{\prime \prime} \otimes\left(\Gamma / \gamma^{\prime}\right) / \gamma^{\prime \prime}$. Of course the logical choice for $\gamma^{\prime \prime}$ is $\gamma / \gamma^{\prime}$ because then $\left(\Gamma / \gamma^{\prime}\right) /\left(\gamma / \gamma^{\prime}\right)=\Gamma / \gamma$.

We only have to prove that $\gamma^{\prime \prime}=\gamma / \gamma^{\prime} \in \Gamma / \gamma^{\prime}$. It is clear that $\gamma / \gamma^{\prime}$ is a subset of internal lines of $\Gamma / \gamma^{\prime}$. Then $\gamma / \gamma^{\prime} \in \Gamma / \gamma^{\prime}$ if $\left.\overline{\operatorname{res}(\gamma}\right) \in R_{V}$ and $\operatorname{res}\left(\gamma^{\prime}\right) \in R_{V}$ implies that $\operatorname{res}\left(\gamma / \gamma^{\prime}\right) \in R_{V}$, which we assumed.

Conversely let us fix $\gamma^{\prime \prime} \in \Gamma / \gamma^{\prime}$ and prove that there exists $\gamma \in \underline{\Gamma}$ containing $\gamma^{\prime}$ such that $\gamma / \gamma^{\prime} \otimes \Gamma / \gamma=\gamma^{\prime \prime} \otimes\left(\overline{\left.\Gamma / \gamma^{\prime}\right)} / \gamma^{\prime \prime}\right.$. Let us write $\gamma^{\prime}=\bigcup_{i \in I} \gamma_{i}^{\prime}$ for the connected components of $\gamma^{\prime}$. Some of these components led to vertices of $\gamma^{\prime \prime}$, the others to vertices of $\left(\Gamma / \gamma^{\prime}\right) \backslash \gamma^{\prime \prime}$. We can then define $\gamma$ as $\left(\gamma^{\prime \prime} \circ_{G_{I_{1}}} \bigcup_{i \in I_{1}} \gamma_{i}^{\prime}\right) \bigcup_{j \in I_{2}} \gamma_{j}^{\prime}$ with $I_{1} \cup I_{2}=I$. It is clearly a subgraph of $\Gamma$ and belongs to $\underline{\Gamma}$ if for all $\gamma_{1}, \gamma_{2} \in \mathscr{H}$ with $\operatorname{res}\left(\gamma_{1}\right) \in R_{V}$, res $\left(\gamma_{2}\right) \in R_{V}$, there exists gluing data $G$ such that $\operatorname{res}\left(\gamma_{1}{ }^{\circ} G \gamma_{2}\right) \in R_{V}$. This is our assumption, so the lemma is proved.

Let us now work out how Lemma 3.2 fits the commutative $\phi^{4}$ model. In this local field theory the divergent graphs have two or four external legs. The residue of a given graph is the one obtained by shrinking all its internal lines to a point (see Section 3.2) and then only depends on the number of external lines of the graph. Let us check condition 2 of Lemma 3.2 for commutative $\phi^{4}$. We consider two graphs $\gamma_{1}$ and $\gamma_{2}$ with two or four external legs. We consider $\gamma_{0}=\gamma_{1}{ }^{\circ} G \gamma_{2}$ for any gluing data $G$. Let $V_{i}, I_{i}$ and $E_{i}$ the respective numbers of vertices, internal and external lines of $\gamma_{i}$, $i \in\{0,1,2\}$. For all $i \in\{0,1,2\}$, we have

$$
\begin{aligned}
4 V_{i} & =2 I_{i}+E_{i}, \\
V_{0} & = \begin{cases}V_{1}+V_{2} & \text { if } E_{2}=2, \\
V_{1}+V_{2}-1 & \text { if } E_{2}=4,\end{cases} \\
I_{0} & = \begin{cases}I_{1}+I_{2}+1 & \text { if } E_{2}=2, \\
I_{1}+I_{2} & \text { if } E_{2}=4,\end{cases}
\end{aligned}
$$

which proves that $E=E_{1}$. Then as soon as $\operatorname{res}\left(\gamma_{1}\right) \in R_{V}$ so does res $\left(\gamma_{0}\right)$. Concerning condition 1 note that $\gamma^{\prime \prime}=\gamma / \gamma^{\prime} \Leftrightarrow$ there exists $G$ such that $\gamma=\gamma^{\prime \prime} \circ_{G} \gamma^{\prime}$, which allows to prove, in the case of a local theory, that condition 1 also holds and that the coproduct (3.15) is coassociative.

Lemma 3.3. Let $H_{c}$ be the linear space of graphs whose residue is $R_{V}$-valued:

$$
\mathscr{H}_{c}=\left\{\Gamma \in \mathscr{H} \mid \operatorname{res}(\Gamma) \in R_{V}\right\} .
$$

$\mathscr{H}_{c}$ is a Hopf subalgebra of $\mathscr{H}$.

Proof. Due to Definition (3.15), $\Delta \mathscr{H}_{c} \subset \mathscr{H}_{c} \otimes \mathscr{H}_{c}$. By induction on the augmentation degree, one also proves that $S\left(\mathscr{H}_{c}\right) \subset \mathscr{H}_{c}$. 


\section{Hopf algebra for non-commutative Feynman graphs}

The definition of the Hopf algebra of non-commutative Feynman graphs which drives the combinatorics of perturbative renormalization is formally the same as in the commutative case [CK00]. But before giving the definitions let us define the residue of a non-commutative graph. As already mentioned, it has been proven (first in [GW05b]) that the Grosse-Wulkenhaar model (2.1) is renormalizable to all orders of perturbation. It means that the divergent parts of the divergent graphs are proportional to mass, wave function, $x^{2}$ and Moyal vertex terms. Following the procedure exposed in Section 3.2, particularly equations (3.13) and (3.14), we find that

$\phi \circ \operatorname{res}(\Gamma)=\delta_{y}(x)+\Delta \delta_{y}(x)+\tilde{x}^{2} \delta_{y}(x)$ if $\Gamma$ is a two-point planar regular graph,

$\phi \circ \operatorname{res}(\Gamma)=\left(\delta_{x_{2}} \star \delta_{x_{3}} \star \delta_{x_{4}}\right)\left(x_{1}\right) \quad$ if $\Gamma$ is a four-point planar regular graph,

which leads to the following graphical definitions:

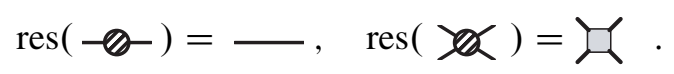

Once more the (graphical) residue of a convergent graph is defined as id $\mathscr{H}$.

Consider now the unital associative algebra $\mathscr{H}$ freely generated by 1PI noncommutative Feynman graphs (including the empty set, which we denote by $\mathbb{1}$ ). The product $m$ is bilinear, commutative and given by the operation of disjoint union. Let the coproduct $\Delta: \mathcal{H} \rightarrow \mathscr{H} \otimes \mathscr{H}$ defined as

$$
\Delta \Gamma=\Gamma \otimes \mathbb{1}+\mathbb{1} \otimes \Gamma+\sum_{\gamma \in \underline{\Gamma}} \gamma \otimes \Gamma / \gamma \quad \text { for all } \Gamma \in \mathcal{H} .
$$

Furthermore let us define the counit $\varepsilon: \mathscr{H} \rightarrow \mathbb{K}:$

$$
\varepsilon(\mathbb{1})=1, \quad \varepsilon(\Gamma)=0 \quad \text { for all } \Gamma \neq \mathbb{1} .
$$

Finally the antipode is given recursively by

$$
S: \mathscr{H} \rightarrow \mathcal{H}, \quad \Gamma \mapsto-\Gamma-\sum_{\gamma \in \underline{\Gamma}} S(\gamma) \Gamma / \gamma .
$$

We can now state our main result.

Theorem 4.1. The quadruple $(\mathcal{H}, \Delta, \varepsilon, S)$ is a Hopf algebra.

Proof. We need only prove the coassociativity of the coproduct (4.2). Then definition (4.4) for the antipode follows from the fact that $\mathscr{H}$ is graded (by the loop number), connected, and from Lemma 3.1.

We will use Lemma 3.2 and the fact that for all $\Gamma \in \mathcal{H}, \operatorname{res}(\Gamma) \in R_{V}$ is equivalent to $\Gamma$ is planar regular. Then conditions 1 and 2 of Lemma 3.2 are equivalent to: 
(1) for all $\gamma$ and $\gamma^{\prime} \subset \gamma$ planar regular, $\gamma / \gamma^{\prime}$ is planar regular;

(2) for all $\gamma$ and $\gamma^{\prime} \subset \gamma$ both planar regular, there exits gluing data $G$ such that $\gamma{ }^{\circ} G \gamma^{\prime}$ is planar regular.

In the following all the graphs we are going to insert will be four-point graphs. The case of two-point graphs is easier and left to the reader. Before proving conditions 1 and 2, let us consider the insertion of a regular four-point graph $\gamma_{2}$ into a vertex of another graph $\gamma_{1}$. Let $\gamma_{0}=\gamma_{1} \circ \gamma_{2}$ and for all $i \in\{0,1,2\}$ let $F_{i}, I_{i}, V_{i}, B_{i}$

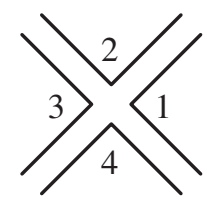

(a) A vertex of $\gamma_{1}$

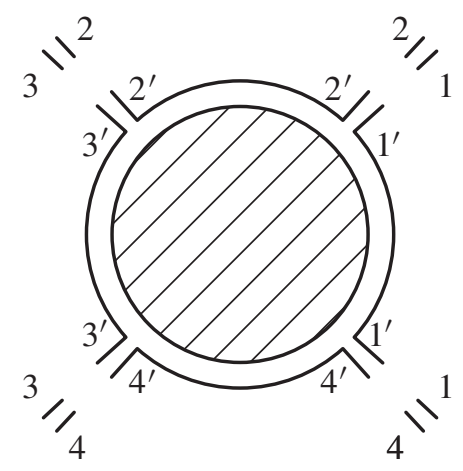

(b) Insertion of $\gamma_{2}$

Figure 3. Insertion procedure.

the respective numbers of faces, internal lines, vertices and broken faces of $\gamma_{i}$. The number of faces of a ribbon graph is the number of closed ${ }^{1}$ single lines. A ribbon vertex is drawn in Figure 3 (a). One sees that the number of faces to which the lines of that vertex belong is at most four. Some of them may indeed belong to the same face. The gluing data necessary for the insertion of $\gamma_{2}$ correspond to a bijection between the half-lines of the vertex in $\gamma_{1}$ and the external lines of $\gamma_{2}$. This last one is regular (only one broken face) and the typical situation is represented in Figure 3 (b). It should be clear that $F=F_{2}-1+F_{1}-n$ for some $n \geqslant 0 . F_{2}-1$ is the number of internal faces of $\gamma_{2}$, i.e., the number of faces of the blob. The number $n$ depends on the gluing data. It vanishes if the insertion respects the cyclic ordering of the vertex. For example, the following bijection $\sigma$ does:

$$
\begin{array}{ll}
\sigma\left(\left(1^{\prime}, 2^{\prime}\right)\right)=(2,3), & \sigma\left(\left(3^{\prime}, 4^{\prime}\right)\right)=(4,1), \\
\sigma\left(\left(2^{\prime}, 3^{\prime}\right)\right)=(3,4), & \sigma\left(\left(4^{\prime}, 1^{\prime}\right)\right)=(1,2) .
\end{array}
$$

As in equations (3.20), $I_{0}=I_{1}+I_{2}$ and $V_{0}=V_{1}+V_{2}-1$. It follows that the genus

\footnotetext{
${ }^{1}$ In the case of external faces, one notices that the corresponding lines are closed.
} 
of $\gamma_{0}$ satisfies

$$
g\left(\gamma_{0}\right)=g\left(\gamma_{1}\right)+g\left(\gamma_{2}\right)+n .
$$

Moreover by exhausting the $4 ! / 4$ possible insertions, one checks that $B_{0} \geqslant B_{1}$. For example, in Figure 4 (a), lines 1 and 3 belong to two different broken faces. Figure 4 (b) shows an insertion of a regular four-point graph which increases the number of broken faces by one: now trajectories $(1,4),(1,2)$ and 3 are external faces (line $(2,4)$ is still an internal one).

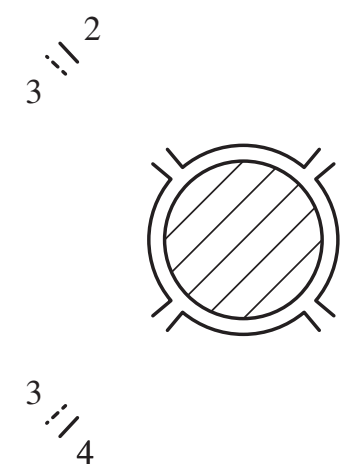

(a) External situation
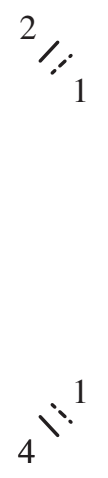

4

Figure 4. Increasing number of external faces.

Let us now turn to proving that the algebra of non-commutative Feynman graphs described above fulfills conditions 1 and 2 .

(1) $\gamma, \gamma^{\prime}$ planar implies $\gamma / \gamma^{\prime}$ planar due to equation (4.6). Furthermore $B(\gamma)=1$ implies that $B\left(\gamma / \gamma^{\prime}\right)=1$ by the preceding remark.

(2) For condition 2 one chooses gluing data $G$ respecting the cyclic ordering of the vertex. Then one has $g\left(\gamma \circ_{G} \gamma^{\prime}\right)=g(\gamma)+g\left(\gamma^{\prime}\right)=0$. The cyclic ordering of the insertion ensures $B\left(\gamma \circ_{G} \gamma^{\prime}\right)=B(\gamma)=1$.

Let $f, g \in \operatorname{Hom}(\mathscr{H}, \mathcal{A}$ ) where $\mathcal{A}$ is the range algebra of the projection $T$ (see Section 3.2). The convolution product $*$ in $\operatorname{Hom}(\mathscr{H}, \mathcal{A})$ is defined by

$$
f * g=m_{\mathscr{A}} \circ(f \otimes g) \circ \Delta_{\mathscr{H}} .
$$

Let $\phi$ be the unrenormalized Feynman rules and $\phi_{-} \in \operatorname{Hom}(\mathscr{H}, \mathcal{A})$ the twisted antipode: for all $\Gamma \in \mathscr{H}$,

$$
\phi_{-}(\Gamma)=-T\left(\phi(\Gamma)+\sum_{\gamma \in \underline{\Gamma}} \phi_{-}(\gamma) \phi(\Gamma / \gamma)\right) .
$$


As in the commutative field theories, the renormalized amplitude $\phi_{+}$of a graph $\Gamma \in \mathscr{H}$ is given by

$$
\phi_{+}(\Gamma)=\phi_{-} * \phi(\Gamma) .
$$

Acknowledgment. We would like to warmly thank Dirk Kreimer for fruitful discussions.

\section{References}

[BGS07] D. N. Blaschke, H. Grosse, and M. Schweda, Noncommutative U(1) gauge theory on $\mathbb{R}_{\Theta}^{4}$ with oscillator term and BRST symmetry. Europhys. Lett. 79 (2007) 61002 .

[CK00] A. Connes and D. Kreimer, Renormalization in quantum field theory and the Riemann-Hilbert problem I: The Hopf algebra structure of graphs and the main theorem. Comm. Math. Phys. 210 (2000), 249-273. Zbl 1032.81026 MR 1748177

[CK01] A. Connes and D. Kreimer, Renormalization in quantum field theory and the Riemann-Hilbert problem II: The $\beta$-function, diffeomorphisms and the renormalization group. Comm. Math. Phys. 216 (2001), 215-241. Zbl 1042.81059 MR 1810779

[DGWW07] A. De Goursac, J.-C. Wallet, and R. Wulkenhaar, Noncommutative induced gauge theory. Eur. Phys. J. C Part. Fields 51 (2007), 977-987.

[DNR01] S. Dăscălescu, C. Năstăsescu, and Ş. Raianu, Hopf algebras. Monogr. Textbooks Pure Appl. Math. 235, Marcel Dekker, New York 2001. Zbl 0962.16026 MR 1786197

[GBV88] J. M. Gracia-Bondía and J. C. Várilly, Algebras of distributions suitable for phasespace quantum mechanics. I. J. Math. Phys. 29 (1988), 869-879. Zbl 0652.46026 MR 940351

[GW03] H. Grosse and R. Wulkenhaar, Renormalisation of $\phi^{4}$-theory on noncommutative $\mathbb{R}^{2}$ in the matrix base. J. High Energy Phys. 12 (2003), 019. MR 2041157

[GW05a] H. Grosse and R. Wulkenhaar, Power-counting theorem for non-local matrix models and renormalisation. Comm. Math. Phys. 254 (2005), 91-127. Zbl 1079.81049 MR 2116739

[GW05b] H. Grosse and R. Wulkenhaar, Renormalisation of $\phi^{4}$-theory on noncommutative $\mathbb{R}^{4}$ in the matrix base. Comm. Math. Phys. 256 (2005), 305-374. Zbl 1075.82005 MR 2160797

[GW07] H. Grosse and M. Wohlgenannt, Induced gauge theory on a noncommutative space. Eur. Phys. J. C Part. Fields 52 (2007), 435-450.

[GMRT07] R. Gurau, A. Malbouisson, V. Rivasseau, and A. Tanasă, Non-commutative complete Mellin representation for Feynman amplitudes. Lett. Math. Phys. 81 (2007), 161-175. MR 2336230 
[GMRVT06] R. Gurau, J. Magnen, V. Rivasseau, and F. Vignes-Tourneret, Renormalization of non-commutative $\Phi_{4}^{4}$ field theory in $x$ space. Comm. Math. Phys. 267 (2006), 515-542. Zbl 1113.81101 MR 2249779

[GR07] R. Gurau and V. Rivasseau, Parametric representation of noncommutative field theory. Comm. Math. Phys. 272 (2007), 811-835. Zbl MR 2304476

[GT07] R. Gurau and A. Tanasă, Dimensional regularization and renormalization of noncommutative QFT. Preprint 2007. arXiv:0706.1147

[Kas95] C. Kassel, Quantum groups. Grad. Texts in Math. 155, Springer-Verlag, New York 1995. Zbl 0808.17003 MR 1321145

[Kre05] D. Kreimer, Structures in Feynman graphs: Hopf algebras and symmetries. In Graphs and patterns in mathematics and theoretical physics, Proc. Sympos. Pure Math. 73, Amer. Math. Soc., Providence, RI, 2005, 43-78. Zbl 1088.81077 MR 2131011

[LSZ04] E. Langmann, R. J. Szabo, and K. Zarembo, Exact solution of quantum field theory on noncommutative phase spaces. J. High Energy Phys. 01 (2004), 017. MR 2045889

[Man03] D. Manchon, Hopf algebras, from basics to applications to renormalization. In Comptes rendus des Rencontres mathématiques de Glanon, Edition 2003. arXiv:math.QA/0408405

[Riv07] V. Rivasseau, Non-commutative renormalization. In Quantum Spaces, Poincaré Seminar 2007, Progr. Math. Phys. 53, Birkhäuser, Basel 2007, 19-107.

[RT07] V. Rivasseau and A. Tanasă. Parametric representation of "critical" noncommutative QFT models. Preprint 2007. arXiv:math-ph/0701034

[VT07] F. Vignes-Tourneret, Renormalization of the orientable non-commutative Gross-Neveu model. Ann. Henri Poincaré 8 (2007), 427-474. Zbl 05172509 MR 2329358

Received July 27, 2007

A. Tanasă, Laboratoire de Physique Théorique, Bât. 210, Université Paris XI, 91405 Orsay Cedex, France, and Dep. Fizica Teoretica, Institutul de Fizica si Inginerie Nucleara H. Hulubei, P. O. Box MG-6, 077125 Bucuresti-Magurele, Romania

E-mail: adrian.tanasa@ens-lyon.org

F. Vignes-Tourneret, IHÉS, Le Bois-Marie, 35 route de Chartres, 91440 Bures-sur-Yvette, France

E-mail: vignes@ihes.fr 